\title{
User Association in Millimeter Wave Cellular Networks with Intelligent Reflecting Surfaces
}

\author{
Ehsan Moeen Taghavi ${ }^{1}$, Alireza Alizadeh $^{2}$, Nandana Rajatheva ${ }^{1}$, \\ Mai $\mathrm{Vu}^{2}$ and Matti Latva-aho ${ }^{1}$ \\ ${ }^{1} 6$ G Flagship, Centre for Wireless Communications, University of Oulu, Oulu, Finland \\ \{seyed.moeentaghavi, nandana.rajatheva, matti.latva-aho\}@oulu.fi \\ ${ }^{2}$ Department of Electrical and Computer Engineering, Tufts University, Medford, USA \\ \{alireza.alizadeh, mai.vu\}@tufts.edu
}

\begin{abstract}
In this paper, we introduce a new load balancing user association scheme for millimeter wave (mmWave) cellular networks in which intelligent reflecting surface (IRS) is applied in the cellular network to improve the coverage region of each cell and mitigate mmWave vulnerability to non-line of sight (N-LoS) paths. The user association scheme improves network performance significantly by adjusting the interference according to the association. We study the IRS-assisted mmWave cellular network where one IRS is deployed to assist in the communication from the base station (BS) to mobile users (MUs) in each cell. We balance BS loads and maximize a network utility by optimizing the user association with a matching game. Simulation results show that the proposed scheme significantly improves the throughput compared to conventional user association techniques.
\end{abstract}

Index Terms-Millimeter wave, User association, Load balancing, Intelligent reflecting surface, Optimization.

\section{INTRODUCTION}

With the pressing need for higher data rates and wider bandwidths, mmWave communications have been considered for the next generation of cellular networks (5G and beyond) due to its capability in providing gigabit data rates and the huge amount of available bandwidth at the range of 30-300 GHz. User association affects the performance of the network and plays a vital role in load balancing of the network by avoiding congestion and providing the required quality of service and lightly-loaded BSs [1].

In mmWave and beyond frequencies, it is hard to build active massive MIMO arrays, and channels at these frequencies are sparse. Intelligent reflecting surface (IRS) is a revolutionary and transformative technology for effectively achieving spectrum and energy efficient wireless communication cost. Precisely, an IRS consists of a large number of low-cost passive elements, each being able to reflect the incident signal independently with an adjustable phase shifter to collaboratively achieve three-dimensional (3D) beamforming without the need of transmit radio-frequency $(\mathrm{RF})$ chains. By small adjustment of phase shifts at each IRS element, the IRS's reflected signals can be added coherently to the desired signal at the receiver, and SINR is improved [2].

It is notable that the IRS is a passive array, and entirely different from other active arrays like relays. Only the ambient signals are reflected by a passive array in IRS, which does not consume any additional power. Furthermore, both direct path and reflected path carry the same information, and therefore they are added coherently [3].

The authors in [4] have studied load balancing user association for single antenna and massive MIMO heterogeneous networks. In their work, user instantaneous rates were independent of user association and active user sets. Most of the time, solving a user association problem is NP-hard. In [5], a heuristic algorithm was proposed to solve the user association problem and achieve a near-optimal solution. Joint problems of user association and resource allocation were investigated in [1], [6]. The most crucial problem in mmWave communications is that its signal is attenuated severely with the increase of the distance between BS and MU. Moreover, there should be a line of sight (LoS) path between BS and MU while in a cellular network, due to the blockage, there might be only the N-LoS path between BS and MU.

In this paper, a new load balancing user association scheme for mmWave MIMO cellular networks is proposed in which IRSs are used to improve the coverage region of each cell and mitigate the mmWave vulnerability to the N-LoS paths. The channel model is assumed as a clustered channel model introduced in [7]. We formulate an optimization problem and a distributed matching algorithm is also proposed to solve the IRS-assisted mmWave cellular network's user association problem.

The rest of the paper is organized as follows: The next section presents the system model. In Sec. III, we 


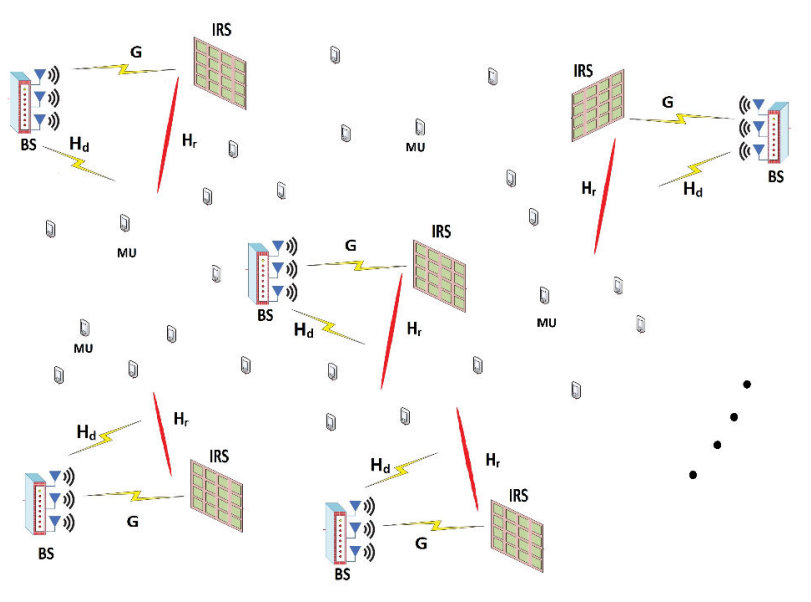

Fig. 1. IRS-assisted mmWave cellular network.

present our proposed user association scenario and its optimization problem. Simulation results are described in Sec. IV, and the conclusion is drawn in Sec. V.

\section{Channel and System Model}

\section{A. mmWave Channel Model}

The mmWave channel model used in this paper is based on the cluster channel model proposed in [7] for urban micro environments which has $C$ clusters with $L$ rays per cluster

$$
H=\frac{1}{\sqrt{C L}} \sum_{c=1}^{C} \sum_{l=1}^{L} \sqrt{\gamma_{c}} a\left(\phi_{c, l}^{M U}, \psi_{c, l}^{M U}\right) a^{*}\left(\phi_{c, l}^{B S}, \psi_{c, l}^{B S}\right),
$$

where $\gamma_{c}$ is the gain of $c^{\text {th }}$ cluster and $\phi_{c, l}^{M U}, \psi_{c, l}^{M U}, \phi_{c, l}^{B S}, \psi_{c, l}^{B S}$ are azimuth and elevation angles of arrival and departure, respectively. $a(\phi, \psi)$ represents the response vector of a uniform planar array (UPA) enabling 3D beamforming in both azimuth and elevation directions. Also, the probability model of LoS and N-LoS considering path loss is utilized. These parameters are generated based on distributions and cross correlations given in [7].

\section{B. Intelligent Reflecting Surface}

In this paper, we consider an IRS-assisted mmWave cellular network, as shown in Fig. 1, where several multi-antenna BSs serve several multi-antenna MUs with the help of several IRSs (e.g., on the wall). Such a cellular network can be employed for indoor applications with a high density of users, e.g., shopping malls, stadiums, and exhibition centers [8]. Since in mmWave communications, the path between the BS and the MU is very vulnerable to the blockage, the cellular network's coverage area is small. The coverage region is extended by assigning an IRS for each cellular network without any additional power consumption. Each user receives the signal from two paths in this scenario: direct link (BS-MU) and reflected link (IRSMU). It is shown that by applying an IRS for each
BS, SINR can be significantly improved compared with conventional cellular networks without IRS [9].

\section{System Model}

A downlink mmWave MIMO cellular network with $J$ BSs and $K$ MUs is considered while $M_{j}$ is the number of antennas at BS $j$, and $N_{k}$ is the number of antennas at MU $k$. Each BS is associated with one IRS with $L_{j}$ phase shifters. The sets of BSs and MUs are denoted by $\mathcal{J}=\{1, \ldots, J\}$ and $\mathcal{K}=\{1, \ldots, K\}$, respectively. Full channel state information (CSI) is considered at both transmitters and receivers. We also define $Q_{j}$ as the Activation Set, which represents the set of active MUs served by the BS $j$. Considering that each MU only receives one data stream, the total number of data streams transmitted by BS $j$ is

$$
D_{j}=\text { Num. of MUs served by BS } j .
$$

The transmitted signal from $\mathrm{BS} j$ is given by

$$
x_{j}=F_{j} d_{j}=\sum_{k \in Q_{j}} f_{k, j} s_{k},
$$

where $s_{k}$ is independent and identically distributed (i.i.d.) random variable with zero mean and unit variance denoted as data for MU $k$. The column vector $d_{j} \in C^{D_{j}}$ is concatenation of $s_{k}, k \in Q_{j}$. It is also notable that $E\left[d_{j} d_{j}^{*}\right]=I_{D_{j}}$ and $f_{k, j} \in C^{M_{j}}$ is beamformer vector designed for each MU $k$ by the $\mathrm{BS} j$ and $F_{j} \in C^{M_{j} \times D_{j}}$ is the total beamformer matrix of the $\mathrm{BS} j$ including all $k \in Q_{j}$.

The power constraint at $\mathrm{BS} j$ can be shown as

$$
E\left[x_{j}^{*} x_{j}\right]=\sum_{k \in Q_{j}} \operatorname{Tr}\left(f_{k, j} f_{k, j}^{*}\right) \leq P_{j} .
$$

As shown in Fig. 1, we consider multi user mmWave cellular network which involves $J$ BSs such that each BS $j$ is assisted by IRS $j$ with $L_{j}$ reflecting passive elements. These IRSs are installed to assist BS/MU communication. Each IRS can adjust the phase shifts of its reflecting passive elements dynamically.

The received signal at MU $k$ can be expressed as

$$
y_{k}=\sum_{j \in \mathcal{J}} H_{k, j}^{*} x_{j}+z_{k}
$$

where $H_{k, j} \in C^{M_{j} \times N_{k}}$ represents the channel matrix between BS $j$ and MU $k$ and $z_{k} \in C^{N_{k}}$ is the white Gaussian noise vector at MU $k$ such that $z_{k} \sim C N\left(0, N_{0} I_{N_{k}}\right)$. It is notable that here, $H_{k, j}$ is a concatenation of three components: BS-IRS link $\left(G_{j} \in C^{M_{j} \times L_{j}}\right)$, IRS reflecting link with phase shifts $\left(H_{r_{k, j}} \in C^{L_{j} \times N_{k}}\right)$, and BS-MU direct link $\left(H_{d_{k, j}} \in C^{M_{j} \times N_{k}}\right)$. Let $\theta_{j}=\left[\theta_{1_{j}}, \theta_{2_{j}}, \ldots, \theta_{L_{j}}\right]$ and $\Theta_{j}=\operatorname{diag}\left(\beta e^{j \theta_{1_{j}}}, \beta e^{j \theta_{2_{j}}}, \ldots, \beta e^{j \theta_{L_{j}}}\right)$ be the diagonal phase-shift matrix where $\theta_{l_{j}} \in[0,2 \pi]$ and $\beta \in[0,1]$ are phase shift and amplitude reflection coefficient 
on the combined incident signal, respectively $\left(l_{j}=\right.$ $\left.\left\{1,2, \ldots, L_{j}\right\}, j=\{1,2, \ldots, J\}\right)$. Therefore, $H_{k, j}$ is as

$$
H_{k, j}=G_{j} \Theta_{j} H_{r_{k, j}}+H_{d_{k, j}} .
$$

Finally, after performing beamforming at the receiver, the processed received signal at MU $k$ is

$$
\tilde{y_{k}}=\sum_{j \in \mathcal{J}} w_{k}^{*} H_{k, j}^{*} x_{j}+w_{k}^{*} z_{k},
$$

where $w_{k} \in C^{N_{k}}$ is the beamformer vector at MU $k$.

\section{User Association and Optimization Problem}

User association is performed on a time slot basis, and each time slot is comparable with channel coherence time in which instantaneous CSI remains the same. Small scale fading characteristics of the channel are assumed to be constant during each transmission. It is assumed that each MU is connected to only one BS. So, the interference structure during each transmission depends on the user association on that transmission.

The association coefficients are defined as $a_{k, j} \in$ $\{0,1\}$ which show the connectivity between $\mathrm{BS} j$ and MU $k$ such that if MU $k$ is associated with BS $j, a_{k, j}=1$ and if there is no association between them $a_{k, j}=0$. As mentioned before, at each transmission, each MU is associated only with one BS. By processing the received signal at each MU, the instantaneous throughput of each user is evaluated. Next, an optimization problem is formulated, and by applying a matching game, the optimal user association is found.

\section{A. Network Interference and Rate Utility}

Network interference highly depends on the structure of the cellular network and user association. Therefore, in user association, besides considering the channel fluctuations, which are very fast in mmWave, interference should be considered. Considering (3) and (7), the received signal at MU $k$ can be expressed as

$$
\begin{aligned}
\tilde{y_{k}}= & \underbrace{w_{k}^{*} H_{k, j}^{*} f_{k, j} s_{k}}_{\text {Desired signal }}+\underbrace{w_{k}^{*} H_{k, j}^{*} \sum_{\substack{i \in Q_{j} \\
\sum_{i j}}} f_{i, j} s_{i}}_{\substack{\text { Intra-cell interference } \\
i \neq k}} \\
& \underbrace{\sum_{i \in Q_{z}} w_{k}^{*} H_{k, z}^{*} f_{i, z} s_{i}+\underbrace{w_{k}^{*} z_{k}}_{\text {Noise }},}_{\text {Inter-cell interference }}
\end{aligned}
$$

where the first term is the desired received signal from $\mathrm{BS} j$, the second term is the interference due to the transmitted signal from BS $j$ intended for the other MUs served by BS $j$, the third term represents the interference sent by the other BSs $(z \neq j)$ for serving MUs associated with them, and the last term is the received noise at MU $k$. As shown in (8), the activation sets $Q_{j}$ and $Q_{z}$ affect the interference terms. So, the network interference depends on the network user association.

During a transmission, when MU $k$ is connected to BS $j$, its instantaneous rate is as follows

$$
\begin{aligned}
R_{k, j}= & \log _{2}\left(1+\zeta_{k, j}^{-1} w_{k}^{*} H_{k, j}^{*} f_{k, j} f_{k, j}^{*} H_{k, j} w_{k}\right), \\
\zeta_{k, j}= & w_{k}^{*} H_{k, j}^{*}\left(\sum_{\substack{i \in Q_{j} \\
i \neq k}} f_{i, j} f_{i, j}^{*}\right) H_{k, j} w_{k} \\
& +w_{k}^{*}\left(\sum_{\substack{z \in \mathcal{J} \\
z \neq j}} \sum_{i \in Q_{z}} H_{k, z}^{*} f_{i, z} f_{i, z}^{*} H_{k, z}\right) w_{k}+N_{0} w_{k}^{*} w_{k} .
\end{aligned}
$$

As shown in (9), the instantaneous rate depends on the user association. Thus, the instantaneous rate for MU $k$ can be presented as

$$
r_{k}=\sum_{j \in \mathcal{J}} a_{k, j} R_{k, j} .
$$

In the mmWave communications, channel varies very fast such that there might be a huge difference in small-scale characteristic of the channel between two consecutive transmission. Therefore, user association must be performed before each transmission. By defining the instantaneous rate vector of the network as $r \triangleq\left(r_{1}, r_{2}, \ldots, r_{k}\right)$, we try to find the optimal association coefficients $\left(a_{k, j}\right)$ and phase shift matrix $\left(\Theta_{j}, j=\right.$ $1,2, \ldots, J)$ which maximizes an overall utility function. The sum rate of the network is proposed as the utility function defined by

$$
U(r) \triangleq \sum_{k \in \mathcal{K}} r_{k}=\sum_{k \in \mathcal{K}} \sum_{j \in \mathcal{J}} a_{k, j} R_{k, j} .
$$

\section{B. Load-Balancing Optimization}

The optimization problem for each transmission can be expressed as

$$
\begin{array}{cl}
\max _{a_{k, j}, \theta_{l_{j}}} . & \sum_{k \in \mathcal{K}} \sum_{j \in \mathcal{J}} a_{k, j} R_{k, j}, \\
\text { s.t. } & \sum_{j \in \mathcal{J}} a_{k, j}=1, \quad \forall k \in \mathcal{K}, \\
& D_{j}=\sum_{k \in \mathcal{K}} a_{k, j} \leq M_{j}, \quad \forall j \in \mathcal{J}, \\
& 0 \leq \theta_{l_{j}} \leq 2 \pi, \quad \begin{array}{l}
l=\{1,2, \ldots, L\}, \\
j=\{1,2, \ldots, J\}, \\
k=\{1,2, \ldots, K\}, \\
j=\{1,2, \ldots, J\}
\end{array},
\end{array}
$$

In the above optimization problem, the first constraint guarantees that all MUs are associated with only one BS, the second constraint is obtained to avoid queuing and congestion at the BSs (the number of MUs served by BS $j$ is limited based on the number of antennas at BS $j$ ). The third constraint represents the bounds on the phase shifts of the IRSs. At each $\mathrm{BS}$, equal power allocation for its own active users is 
considered. Hence the power constraint in (4) can be neglected. We also assume that full CSI at both BSs and MUs is available. By this assumption, after solving (12), an upper bound of the sum rate is provided.

It is assumed that in the above optimization problem, at first the phase shifters are optimized such that $\arg \left(G_{j} \Theta_{j} H_{r_{k, j}}\right)=\arg \left(H_{d_{k, j}}\right)$ in order the reflected signals from the IRS are added to the desired signals from the direct link coherently [10]. After obtaining phase shift arrays at the IRSs, singular value decomposition (SVD) is used to obtain beamformers at the BSs $\left(f_{k, j}\right)$ and the MUs $\left(w_{k}\right)$ [5]. It is notable that considering SVD-precoding, an upper bound of performance is provided. Moreover, the precoding index (vector) can be sent to MUs by BSs/CRAN.

\section{Matching game solution}

The optimization problem in (12) is an integer nonlinear programming known to be NP-hard due to its nonlinear structure and presence of integer variables. Since the utility function in (12) is neither convex nor concave, it is challenging to solve analytically. Thus we propose a heuristic algorithm based on matching theory to obtain a solution. In this paper, the user association problem is formulated as a distributed matching game between the BSs and the MUs, and Deferred Acceptance matching game (DA-MG) is used to associate MUs with BSs [11]. In order to formulate the user association as a matching game, some definitions are introduced as below:

Definition 1. Each $M U k$ and $B S j$ builds a preference relation $\left(\geq_{k}\right.$ and $\left.\geq_{j}\right)$ between each pair of BSs and MUs respectively based on the instantaneous user rates.

Therefore, for any two MUs $k, l \in \mathcal{K}, k \neq l$ each BS builds a preference relation $\geq_{j}$ such that

$$
k \geq_{j} l \Leftrightarrow \Psi_{k, j}^{B S} \geq \Psi_{l, j}^{B S} \Leftrightarrow \text { BS } j \text { prefers MU } k \text { to MU } l \text {. }
$$

where $\Psi^{B S}$ is the objective function of BSs, and $\Psi_{k, j}^{B S}$ is the value of the objective function when BS $j$ serves MU $k$. Similarly, for any two BSs $i, j \in \mathcal{J}, i \neq j$ :

$$
j \geq_{k} i \Leftrightarrow \Psi_{k, j}^{M U} \geq \Psi_{k, i}^{M U} \Leftrightarrow \text { MU } k \text { prefers BS } j \text { to BS } i .
$$

where $\Psi^{M U}$ is the objective function of MUs.

Each MU and BS builds its desired preference list in descending order of interest over the set of BSs and MUs, respectively based on the preference relations. The length of each MU and BS's preference list is $J$ and $K$, respectively. Considering the preference list of each MU and each BS, the preference matrix $P_{\mathcal{K}}$ of size $K \times J$ and the preference matrix $P_{\mathcal{J}}$ of size $J \times K$ are built for the MUs and the BSs respectively.

Definition 2. The tuple $\left(\mathcal{J}, \mathcal{K}, P_{\mathcal{J}}, P_{\mathcal{K}}, \mathcal{D}_{\mathcal{J}}, \mathcal{B}_{\mathcal{K}}\right)$ is defined as the user association matching game (MG) where $\mathcal{D}_{\mathcal{J}}=\left[D_{1}, D_{2}, \ldots, D_{J}\right]$ is the vector of BSs' quotas

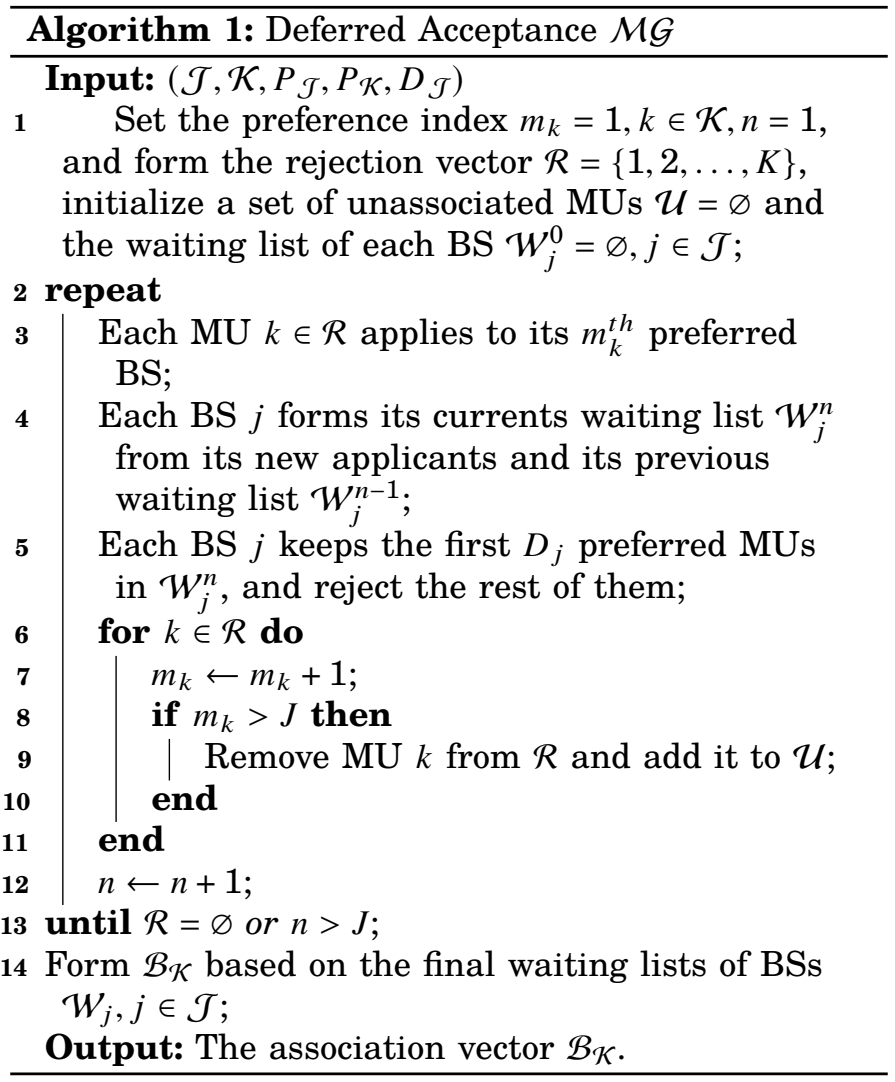

and $\mathcal{B}_{\mathcal{K}}=\left[\beta_{1}, \beta_{2}, \ldots, \beta_{K}\right]$ is the association vector as the outcome of the game, and $\beta_{k}, k \in \mathcal{K}$ is the association factor of $M U k$ and represents the index of BS to whom user $k$ is associated, i.e., $\beta_{k} \in \mathcal{J}$ with $k \in \mathcal{K}$.

In a matching game user association, MUs and BSs are the game players which can have their own objective functions and build their preference lists individually. In our proposed matching game, the user instantaneous rate in (9) is considered as the objective function for both sides of the game (MUs and BSs), i.e., $\Psi_{k, j}^{B S}=\Psi_{k, j}^{M U}=R_{k, j}$. This objective function only depends on the SINR computed at each MUs and reported to the network through the physical uplink shared channel (PUSCH) or physical uplink control channel (PUCCH). Therefore, the proposed matching game is fast and suitable for the $5 \mathrm{G}$ and beyond networks include URLLC (ultra reliable low latency communications).

In this paper, we employ DA-MG to solve the user association problem. The main inputs of this game are the preference lists of MUs and BSs, and its output is a load balanced association vector $\mathcal{B}_{\mathcal{K}}$. Initially, we define $m_{k}=1$ as the preference index of each MU $k$, build an initial rejection set $\mathcal{R}=\{1,2, \ldots, K\}$, and initialize $\mathcal{U}=\varnothing$ as the set of unassociated MUs and $\mathcal{W}_{j}^{0}=\varnothing$ as the waiting list of each BS $j$. At the beginning of the game, BSs and MUs build their preference lists based 


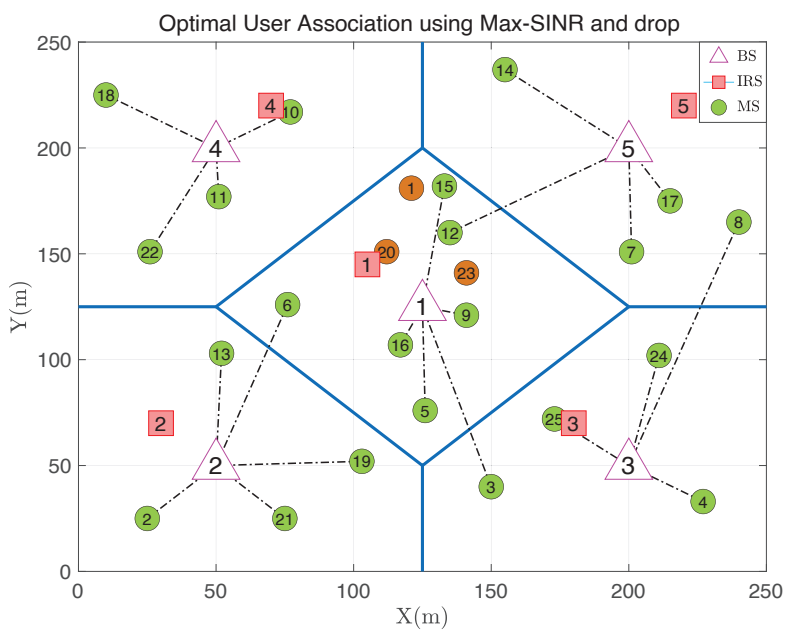

Fig. 2. User association in IRS-assisted mmWave cellular network: the conventional max-SINR and drop scheme [5].

on data rates (SINR) measured at MUs and report back to BSs. At each iteration, each MU applies to its $m_{k}^{\text {th }}$ preferred BS according to its preference list. Next, each BS $j$ ranks all its new applicants and the applicants in its previous waiting list based on its preference list. The first $D_{j}$ MUs form the new waiting list of the $\mathrm{BS}$, and the rest of them are rejected (transferred to $\mathcal{R})$. The rejected MUs update their preference index $\left(m_{k} \leftarrow m_{k}+1\right)$, and apply to their next preferred BS in the next iteration of the game. The game iterates until either the rejection set is empty or all rejected users have applied to all BSs [11]. At this point, each BS performs association with all users in its waiting list; any user left in the rejected set is unassociated. A summary of the DA matching game is described in Algorithm 1.

\section{Simulation Results}

In this section, the proposed user association scheme's performance in IRS-assisted mmWave cellular network is analyzed. We consider a mmWave network operating at $28 \mathrm{GHz}$ with $5 \mathrm{BSs}(J=5), 5$ IRSs (each IRS is associated with one BS), and 25 MUs $(K=25)$. Each BS can serve 5 MUs simultaneously $\left(D_{j}=5, j=1, \ldots, 5\right)$. The mmWave links are generated as described in Sec. II such that each link involves 5 clusters with 10 rays per cluster. Each BS is equipped with an $8 \times 8 \mathrm{UPA}$ of antennas, each IRS is equipped with $256 \times 256$ arrays (it is notable that in order for the indirect path to be useful, the number of passive elements at IRS should be large enough) and each MU is equipped with a $4 \times 1$ ULA of antennas. Noise power spectral density is $-127 \mathrm{dBm} / \mathrm{Hz}$ and all BSs transmit with equal power $P_{j}$. BSs are located at specific locations, IRSs are located randomly around $\mathrm{BSs}$, and MUs are distributed randomly in a region size of $250 \mathrm{~m} \times 250 \mathrm{~m}$. For the IRS to be useful, an LoS

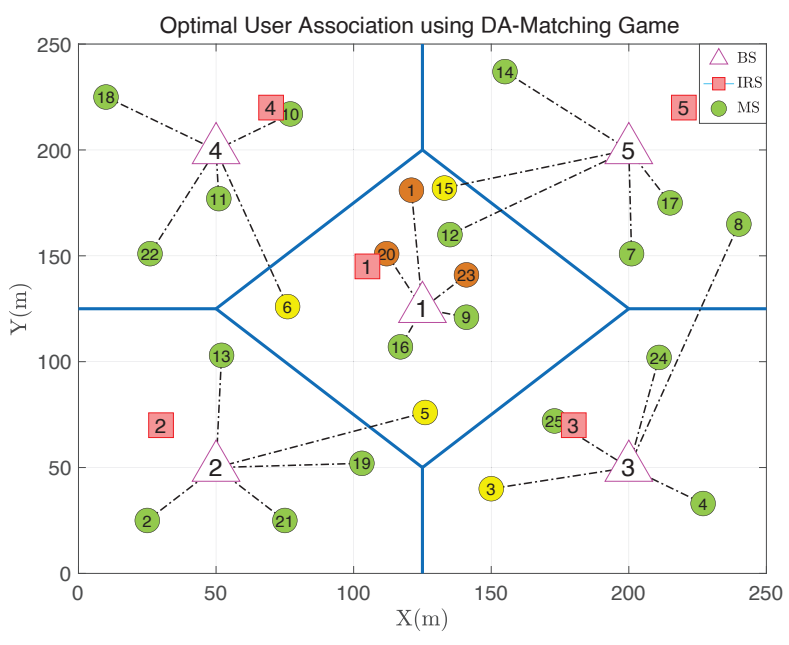

Fig. 3. User association in IRS-assisted mmWave cellular network: the proposed DA-MG scheme.

path between each BS and its corresponding IRS is considered.

Figs. 2 \& 3 show user association in an IRS-assisted mmWave cellular network by the conventional maxSINR and drop user association scheme [5] and the DA-MG association scheme, respectively. In the first scheme, each MU aimed to associate with the BS providing the highest SINR, and the congested BSs drop the overloading MUs, since each BS $j$ cannot serve more than $D_{j}$ MUs. However, in the second scheme, the MUs are associated with BSs according to BSs' load constraints. As shown in Fig. 2, some MUs are not associated (MUs \#1, \#20, and \#23), while some BSs are underloaded (BSs \#3, \#4, and \#5), resulting in poor fairness and quality of service. It can be seen from Fig. 3 that by applying the proposed association technique, the BSs' loads are balanced in such a way that while the maximum sum rate in the cellular network is achieved, all the MUs are served and no congestion occurs in the BSs by pushing the overloading MUs from the congested BSs into the lightly-loaded BSs. Moreover, to mitigate the interference and N-LoS problem, the MUs are assigned to the most appropriate BS in the cellular network regardless of the MU-BS distance. Therefore, MUs \#3, \#5, \#6, and \#15 are assigned to other BSs compared to Fig. 2.

The performance of the proposed algorithm and the IRS's impact in the mmWave cellular network are shown in Fig. 4. Spectral efficiency of mmWave cellular network (with and without the assistance of IRS) versus the BSs' transmit power for the proposed scheme is depicted in Fig. 4. As shown in the figure, IRS is mostly useful at mmWave frequencies where the channels are sparse, and there might be only N-LoS link between the BS and MU. Thus, an extra propagation path through IRS is essential even if this path is weak due to channel attenuation at this band. As shown in the Fig. 4, the 


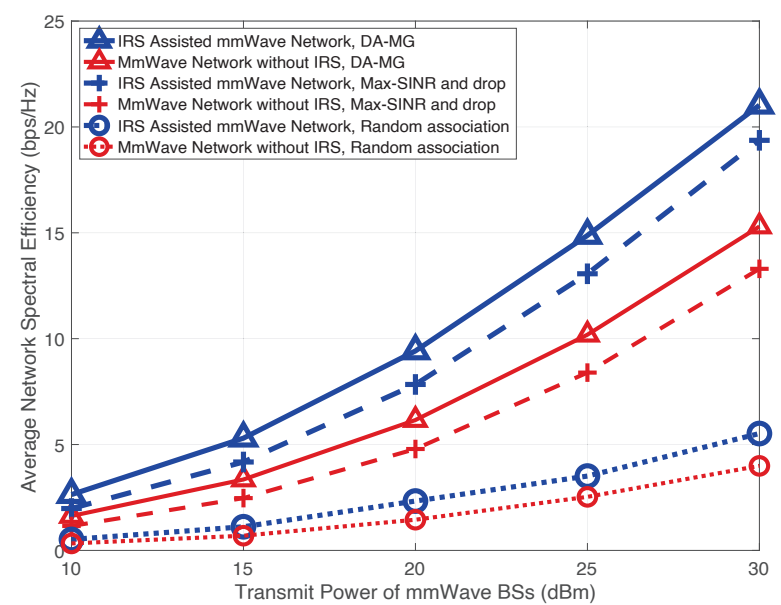

Fig. 4. Average network spectral efficiency with and without IRS assistance.

spectral efficiency performance of the proposed user association scheme outperforms in comparison with two other user association schemes: (i) max-SINR user association with user drop [5] and (ii) random user association. For the second scheme, which is the worst, MUs are assigned randomly to the BSs according to load balancing constraints.

Fig. 5 depicts the cumulative distribution function (CDF) of users' spectral efficiency for mmWave cellular network with and without the assistance of the IRS. It can be observed that the probability of having lower spectral efficiency is higher for the network without the IRS, which shows the advantage of using IRSs, especially when there is no LoS path between BSs and MUs. By focusing on the figure's low rate region, it can be inferred that compared to the max-SINR and drop scheme and random association scheme, the DA-MG scheme shows a lower probability of users having an extremely low data rate. For example, the probability of having users with a data rate smaller than $0.01 \mathrm{bps} / \mathrm{Hz}$ in an IRS-assisted network is less than $1 \%$ for the DA-MG scheme while this probability is $15 \%$ for maxSINR and drop scheme and $40 \%$ for random association scheme. Therefore, the DA-MG scheme enhances fairness for MUs.

\section{Conclusion}

In this paper, we considered an IRS-assisted mmWave cellular network and formulated the problem of optimal user association considering the impact of applying IRS for spectral efficiency improvement. Using a matching game for assigning the MUs to BSs, the network's aggregated interference was mitigated, and the network sum-rate increased. At the same time, no congestion occurred in the BSs. Simulation results confirmed the fact that since the channel gain in mmWave is very vulnerable to the path loss and the N-LoS path, by applying an IRS cooperated with the BS in each cell,

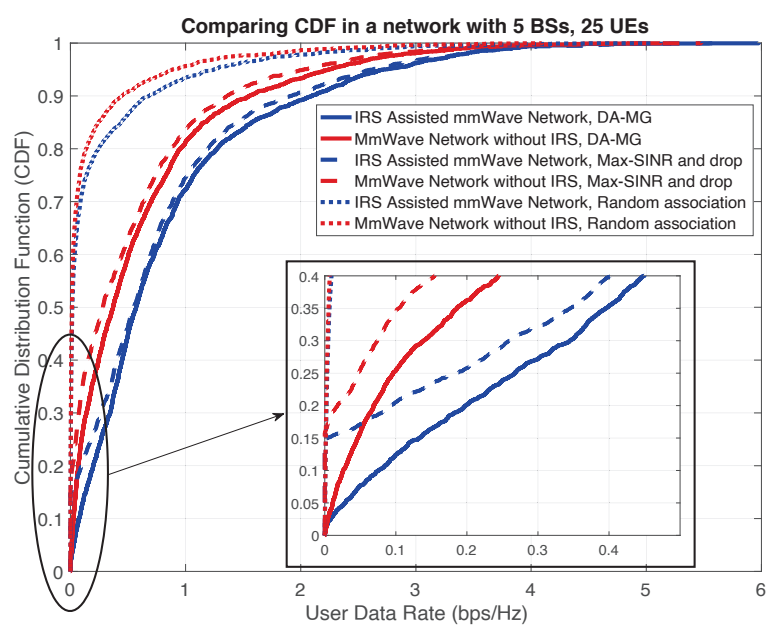

Fig. 5. CDF of users' spectral efficiency, showing significantly higher probability of higher rates for cell edge users using IRS.

an extra reflected propagation path is added coherently to the direct path and hence, the spectral efficiency of the network is increased significantly.

\section{REFERENCES}

[1] G. Dong, H. Zhang, S. Jin and D. Yuan, "Energy-EfficiencyOriented Joint User Association and Power Allocation in Distributed Massive MIMO Systems," in IEEE Transactions on Vehicular Technology, vol. 68, no. 6, pp. 5794-5808, June 2019.

[2] Q. Wu and R. Zhang, "Intelligent Reflecting Surface Enhanced Wireless Network via Joint Active and Passive Beamforming," in IEEE Transactions on Wireless Communications, vol. 18, no. 11, pp. 5394-5409, Nov. 2019.

[3] C. Huang, A. Zappone, G. C. Alexandropoulos, M. Debbah and C. Yuen, "Reconfigurable Intelligent Surfaces for Energy Efficiency in Wireless Communication," in IEEE Transactions on Wireless Communications, vol. 18, no. 8, pp. 4157-4170, Aug. 2019.

[4] D. Bethanabhotla, O. Y. Bursalioglu, H. C. Papadopoulos and G. Caire, "Optimal User-Cell Association for Massive MIMO Wireless Networks," in IEEE Transactions on Wireless Communications, vol. 15, no. 3, pp. 1835-1850, March 2016.

[5] A. Alizadeh and M. Vu, "Load Balancing User Association in Millimeter Wave MIMO Networks," in IEEE Transactions on Wireless Communications, vol. 18, no. 6, pp. 2932-2945, June 2019.

[6] X. Qin, X. Yuan, Z. Zhang, F. Tian, Y. T. Hou and W. Lou, "Joint User-AP Association and Resource Allocation in Multi-AP 60GHz WLAN," in IEEE Transactions on Vehicular Technology, vol. 68, no. 6, pp. 5696-5710, June 2019.

[7] 3rd Generation Partnership Project (3GPP), "Study on channel model for frequencies from 0.5 to $100 \mathrm{GHz}$," Technical Report 38.901, Jun. 2018, v. 15.0.0.

[8] C. Pan, H. Ren, K. Wang, W. Xu, M. Elkashlan, A. Nallanathan and L. Hanzo, "Multicell MIMO Communications Relying on Intelligent Reflecting Surfaces" in IEEE Transactions on Wireless Communications, vol. 19, no. 8, pp. 5218-5233, 2020.

[9] Q. Wu and R. Zhang, "Beamforming optimization for wireless network aided by intelligent reflecting surface with discrete phase shifts", in IEEE Transactions on Communications, vol. 68 , no. 3, pp. $1838-1851,2020$.

[10] E. Björnson, Ö. Özdogan and E. G. Larsson, "Intelligent Reflecting Surface Versus Decode-and-Forward: How Large Surfaces are Needed to Beat Relaying?," in IEEE Wireless Communications Letters, vol. 9, no. 2, pp. 244-248, Feb. 2020.

[11] A. Alizadeh and M. Vu, "Early Acceptance Matching Game for User Association in 5G Cellular HetNets," 2019 IEEE Global Communications Conference (GLOBECOM), Waikoloa, HI, USA, 2019, pp. 1-6. 